Rechtsmedizin 2021 · 31:35-41 https://doi.org/10.1007/s00194-020-00444-7 Online publiziert: 13 . November 2020 (c) Der/die Autor(en) 2020

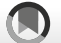

Check for
updates

M. Timme' $\cdot$ A. Karch ${ }^{2} \cdot$ D. Shay ${ }^{2} \cdot$ C. Ottow ${ }^{3} \cdot$ A. Schmeling

'Institut für Rechtsmedizin, Universitätsklinikum Münster, Westfälische Wilhelms-Universität Münster, Münster, Deutschland

${ }^{2}$ Institut für Epidemiologie und Sozialmedizin, Universitätsklinikum Münster, Westfälische WilhelmsUniversität Münster, Münster, Deutschland

${ }^{3}$ Klinik für Radiologie, Universitätsklinikum Münster, Westfälische Wilhelms-Universität Münster, Münster, Deutschland

\title{
Zur Altersdiagnostik lebender Personen: der Einfluss des sozioökonomischen Status auf die Skelett- und Zahnentwicklung in einer deutschen Studienkohorte
}

zu die Entwicklung des Handskeletts im Röntgenbild, die Entwicklung der dritten Molaren in der Panoramaschichtaufnahme (PSA bzw. OPG) und, bei radiologisch abgeschlossener Entwicklung des Handskeletts, die Entwicklung der medialen Schlüsselbeinepiphysen in der Computertomographie (CT) herangezogen $[4,10,11]$.

Soll das Überschreiten juristisch relevanter Altersgrenzen mit dem höchsten Beweismaß bewertet werden, ist bei der Beurteilung das niedrigste Alter maßgeblich, in dem die festgestellte Merkmalsausprägung, also der jeweilige Grad der Entwicklung, wie er bei der untersuchten Person vorliegt, in einer Referenzpopulation nachgewiesen werden konnte. Da mehrere Merkmalssysteme bei der Altersdiagnostik berücksichtigt werden, ist das höchste für die jeweilige Untersuchung festgestellte Mindestalter anzunehmen. Durch dieses „Mindestalterkonzept " ist es nahezu ausgeschlossen, dass das forensische Alter der untersuchten Person zu hoch angegeben wird [4].

Nachdem in den vergangenen Jahren ein Schwerpunkt auf der Verfeinerung der Methodik wie der Stadiensysteme der Merkmalsausprägungen, der Technik der Bildgebung sowie der Generierung von
Referenzwerten lag, ist es weiterhin von Interesse, welche Faktoren die Entwicklung der untersuchten Merkmale beeinflussen können [3, 7, 12]. Dies ist zum einen von Bedeutung, damit im Einzelfall Personen mit einer besonderen Beeinflussung der Merkmalssysteme von der Altersbegutachtung ausgeschlossen werden können, wenn ihre spezielle Situation nicht in der Referenzpopulation abgebildet ist. Zum anderen ist eine genaue Kenntnis über die beeinflussenden Faktoren von Bedeutung, damit diese bei der Zusammensetzung von Referenzpopulationen bzw. bei der konkreten Auswahl der im Einzelfall heranzuziehenden Referenzwerte berücksichtigt werden können.

Ein möglicher Einflussfaktor auf die Entwicklung ist beispielsweise der Ernährungsstatus. Timme et al. untersuchten in einer aktuellen Studie den Einfluss des Body-Mass-Index (BMI) als Indikator für den Ernährungsstatus auf die Skelett- und Zahnentwicklung und konnten dabei keinen Zusammenhang zwischen dem Ernährungsstatus und der Zahnentwicklung nachweisen. Die Autoren unterstreichen dabei die Bedeutung der Untersuchung weiterer möglicher Einfluss- diagnostik (AGFAD) der Deutschen Gesellschaft für Rechtsmedizin werden da- 
Tab. 1 Zusammensetzung der Studienpopulation nach Alter und Geschlecht; Fälle insgesamt und tatsächlich ausgewertete Fälle (tatsächlich ausgewertet/insgesamt)

\begin{tabular}{llll}
\hline Altersgruppe (Jahre) & Weiblich $(\boldsymbol{n})$ & Männlich $(\boldsymbol{n})$ & Summe $(\boldsymbol{n})$ \\
\hline 12 & $16 / 16$ & $21 / 21$ & $37 / 37$ \\
\hline 13 & $13 / 14$ & $27 / 29$ & $40 / 43$ \\
\hline 14 & $24 / 26$ & $18 / 20$ & $42 / 46$ \\
15 & $27 / 27$ & $23 / 25$ & $50 / 52$ \\
16 & $29 / 29$ & $25 / 26$ & $54 / 55$ \\
17 & $23 / 29$ & $25 / 26$ & $48 / 55$ \\
18 & $27 / 28$ & $26 / 29$ & $53 / 57$ \\
19 & $29 / 29$ & $24 / 25$ & $53 / 54$ \\
\hline 20 & $22 / 27$ & $22 / 25$ & $44 / 52$ \\
21 & $24 / 29$ & $25 / 28$ & $49 / 57$ \\
22 & $22 / 29$ & $24 / 27$ & $46 / 56$ \\
23 & $19 / 28$ & $9 / 27$ & $28 / 55$ \\
24 & $19 / 24$ & $18 / 27$ & $37 / 51$ \\
\hline Summe & $294 / 335$ & $287 / 335$ & $581 / 670$
\end{tabular}

faktoren auf die Merkmale der forensischen Altersdiagnostik [13].

Beim Skelettalter wird davon ausgegangen, dass Populationsunterschiede nicht auf genetische Faktoren zurückzuführen sind, sondern vom sozioökonomischen Status (SES) einer Population abhängen. Dabei geht ein vergleichsweise höherer SES mit einem fortgeschrittenen Skelettalter einher [14]. Dieser Zusammenhang wurde in verschiedenen, geografisch abgrenzbaren Kollektiven und innerhalb von regionalen Kollektiven beschrieben [14, 15].

Für die Zahnentwicklung wird angenommen, dass sie weitgehend unabhängig von äußeren Einflüssen abläuft und im Wesentlichen genetisch determiniert ist [16-19]. Eine Diskrepanz zwischen Skelettalter und Zahnalter der zu begutachtenden Person kann demzufolge darauf hinweisen, dass Skelettreifungsstörungen, z. B. verursacht durch endokrine Störungen, vorliegen [4].

In dieser Studie sollte der Einfluss des SES auf die Skelettentwicklung sowie auf die Zahnentwicklung innerhalb eines lokalen Kollektivs untersucht werden. Die Ausgangshypothese war, dass ein vergleichsweiser höherer SES mit einer fortgeschrittenen Skelettentwicklung einhergeht. Außerdem wurde angenommen, dass die Zahnentwicklung unabhängig vom SES ist.

\section{Material und Methode}

Für die vorliegende Studie wurden insgesamt 670 Freiwillige im Alter von 12 bis 24 Jahren prospektiv untersucht. Dabei handelte es sich um 335 weibliche und 335 männliche Teilnehmer. Die Zusammensetzung der Studienpopulation nach Alter und Geschlecht sowie die tatsächlich im Rahmen des Regressionsanalysen ausgewerteten Fälle zeigt $\bullet$ Tab. 1. Die Altersgruppen umfassten immer volle Jahre, d.h., dass in der Altersgruppe „24 Jahre“ Personen im Altervon 24,00 bis 24,99 Jahren zusammengefasst wurden. Das Alter sowie ein Geburtsort innerhalb Deutschlands mussten durch offizielle Dokumente belegt werden. Nach umfassender Aufklärung wurde von allen Teilnehmern eine schriftliche Einverständniserklärung eingeholt - für die minderjährigen Teilnehmer musste außerdem das schriftliche Einverständnis der Eltern vorliegen. Für diese Studie lag ein positives Votum der zuständigen Ethikkommission vor.

Die Aufnahmen mit dem Magnetresonanztomographen (MRT) (Philips 3,0 T Achieva; Fa. Philips Medical Systems, Hamburg, Deutschland) wurden im Translational Imaging Center (TRIC) der Klinik für Radiologie des Universitätsklinikums Münster durchgeführt. Die Befundung der Bilder erfolgte an einem abgedunkelten Arbeitsplatz (View Forum Workstation; Fa. Philips Medical Systems, Hamburg, Deutschland).
Für das Skelettalter wurden folgende Regionen ausgewertet: die mediale Klavikulaepiphysenfuge, die proximale Epiphysenfuge der Tibia, die distale Epiphysenfuge des Femurs, die distale Epiphysenfuge des Radius. Der Ossifikationsgrad der Epiphysenfugen wurde mithilfe der Stadieneinteilungen nach Schmeling et al. (2004) [20] und Kellinghaus et al. (2010) [21] bestimmt.

Die Zahnentwicklung wurde jeweils am linken unteren dritten Molaren bewertet. Dazu wurde dem Zahn jeweils ein Entwicklungsstadium nach Demirjian (1973) [22] in der Modifikation von Mincer et al. (1993) [23] zugeordnet.

Darüber hinaus wurde der sozioökonomische Status (SES) der Teilnehmer erfasst. Dazu wurde der Bildungsgrad der Eltern erfragt. Es wurden folgende Bildungsgrade unterschieden, welche sich an der International Standard Classification of Education (ISCED) der Organisation der Vereinten Nationen für Erziehung, Wissenschaft und Kultur (UNESCO) orientierten [24]: kein Schulabschluss (1), Hauptschulabschluss (2), Realschulabschluss (3), Abitur (4), abgeschlossene Berufsausbildung (5), abgeschlossenes Fachhochschulstudium (6), abgeschlossenes Universitätsstudium (7), Promotion (8), Habilitation (9). Zur Auswertung wurden die Bildungsgrade in 3 Stufen zusammengefasst, wobei sich an den Empfehlungen der Deutschen Gesellschaft für Epidemiologie zur Messung soziodemografischer Merkmale in epidemiologischen Studien orientiert wurde, welche die Differenzierung von 3 Statusgruppen (Leveln) vorschlagen [25]: Level 1 (niedriges Bildungslevel): Bildungsgrade 1-4; Level 2 (mittleres Bildungslevel): Bildungsgrade $5+6$, Level 3 (hohes Bildungslevel): Bildungsgrade 7-9.

Der Zusammenhang zwischen den untersuchten Merkmalen und dem Bildungsniveau der Eltern wurde mithilfe von Regressionsanalysen untersucht. Dabei wurden das höchste Bildungsniveau der Eltern sowie das additiv kombinierte Bildungsniveau beider Eltern berücksichtigt. Außerdem wurde der Einfluss auf den Gesamtentwicklungsgrad des Skeletts (alle ossären Merkmale ohne Zahnentwicklung) untersucht. 
Für die Ergebnisse der linearen Regressionsanalysen wurden der Regressionskoeffizient ( $\beta$ ) mit $95 \%$-Konfidenzintervall (KI), das Bestimmtheitsmaß $\left(\mathrm{r}^{2}\right)$ sowie die $p$-Werte angegeben.

\section{Ergebnisse}

Bei einigen Teilnehmern konnten nicht alle Skelettregionen untersucht werden, da die Untersuchungen im Magnetresonanztomographen (MRT) wegen Unwohlsein oder anhaltender Bewegung abgebrochen werden mussten (• Tab. 1). Dass bei 287 männlichen und bei 294 weiblichen Individuen alle Daten erhoben werden konnten (87\%), zeigt - Tab. 1. Diese Fälle bilden letztlich die Grundlage für die Regressionsanalysen. Die Zusammensetzung der Studienpopulation nach sozioökonomischem Status (SES) und Entwicklungsstadien der untersuchten Merkmale zeigt • Tab. 2.

Das Ausbildungsniveau der Mütter der Teilnehmer reichte vom Hauptschulabschluss $(n=7)$ bis zur Habilitation $(n=2)$. Bei den Vätern der Teilnehmer war ein Ausbildungsniveau von „kein Schulabschluss" $(n=1)$, Hauptschulabschluss $(n=8)$ bis zur Habilitation $(n=11)$ vertreten. Insgesamt hatten bei den Müttern der Teilnehmer 5,5\% ein niedriges, 60,5\% ein mittleres und 33,9\% ein hohes Ausbildungslevel. Für die Väter lagen die entsprechenden Werte bei $4,5 \%, 48,7 \%$ bzw. $46,8 \%$ (• Tab. 2). Insgesamt unterschied sich das Ausbildungslevel der Eltern der Teilnehmer nicht zwischen deren Geschlechtern $(p=0,52$ bzw. $p=0,96)$.

Die Ossifikation war mit einem mittleren Stadium von 3c bzw. 4 für Tibia, Femur und Radius insgesamt weit fortgeschritten. Für die mediale Klavikulaepiphysenfuge lag der mittlere Entwicklungsgrad bei 3a. Die Weisheitszähne waren bei beiden Geschlechtern im Mittel entsprechend einem Grad F nach Demirjian (1972) entwickelt.

Die Ergebnisse der Regressionsanalysen zeigt - Tab. 3. Es konnte für keine Untersuchung ein Zusammenhang zwischen SES und den evaluierten Merkmalen festgestellt werden.

Das Bestimmtheitsmaß als Maß für die globale Anpassungsgüte und somit

Rechtsmedizin 2021 · 31:35-41 https://doi.org/10.1007/s00194-020-00444-7

(c) Der/die Autor(en) 2020

M. Timme · A. Karch · D. Shay · C. Ottow · A. Schmeling

\section{Zur Altersdiagnostik lebender Personen: der Einfluss des sozioökonomischen Status auf die Skelett- und Zahnentwicklung in einer deutschen Studienkohorte}

\section{Zusammenfassung}

Die forensische Altersdiagnostik kann ein entscheidendes Instrument zur Durchführung rechtstaatlicher Verfahren sein. Bei der Altersdiagnostik wird die Entwicklung von Skelett- und Zahnmerkmalen einer zu begutachtenden Person mit Daten von Referenzpopulationen verglichen. Als möglicher Einflussfaktor auf die Altersdiagnose ist der sozioökonomische Status (SES) der zu begutachtenden Person zu berücksichtigen. Allerdings ist die Studienlage zu diesem Einflussfaktor bislang spärlich. Prospektiv wurde der Zusammenhang zwischen der Skelett- bzw. Zahnentwicklung und dem SES innerhalb einer deutschen Studienpopulation aus freiwilligen Teilnehmern ermittelt. Dazu wurde die Entwicklung der medialen Epiphyse des Schlüsselbeins, der distalen Epiphyse des Radius, der distalen Epiphyse des Femurs, der proximalen Epiphyse der Tibia und des linken unteren dritten Molaren bei 294 Frauen und
287 Männern im Alter von 12 bis 24 Jahren mittels 3-Tesla-Magnetresonanztomographie (MRT) untersucht. Zusätzlich wurde der SES der Teilnehmer erfasst. Hierzu wurde das Bildungsniveau der Eltern erfragt. Der Einfluss des SES auf die Einzelmerkmale sowie die Gesamtheit der untersuchten Skelettmerkmale wurde mittels linearer Regression ermittelt. Für alle untersuchten Merkmale und Kombinationen konnte keine Evidenz für einen Zusammenhang festgestellt werden. Es wurde geschlussfolgert, dass sich die Lebensumstände der Studienteilnehmer offenbar nicht ausreichend stark unterschieden, um einen Einfluss des SES auf das Skelettalter darzustellen.

\section{Schlüsselwörter}

Forensische Altersschätzung · Sozialer Status . Skelettalter · Dentales Alter

\section{Age assessment of living individuals: the influence of socioeconomic status on skeletal and dental development in a German study cohort}

Abstract

Forensic age assessment can be a powerful lever to ensure the rule of law. In age assessment procedures, the development of skeletal and dental features is compared with reference populations. The socioeconomic status (SES) of the person to be assessed is to be considered as a possible influencing factor on the assessment of age; however, the available studies on this influencing factor are scarce so far. Prospectively, the relationship between skeletal or dental development and SES was determined within a German study population of volunteers. For this purpose the development of the medial epiphysis of the clavicle, the distal epiphysis of the radius, the distal epiphysis of the femur, the proximal epiphysis of the tibia and the left lower third molar was examined in 294 females and 287 males aged between
12 and 24 years using magnetic resonance imaging (MRI) at $3 \mathrm{~T}$. Additionally, the SES of all persons was determined. For this, the education level of the parents of the persons examined was queried. The influence of the SES on the individual characteristics as well as on the totality of the examined skeletal characteristics was determined by linear regression. No evidence for a relationship could be found for all characteristics and combinations. It was concluded that the life circumstances of the study participants apparently did not differ sufficiently to demonstrate an influence of the SES on skeletal age.

\section{Keywords}

Forensic age estimation - Social standing . Skeletal age - Dental age 


\section{Originalien}

Tab. 2 Charakteristika der untersuchten Studienpopulation $(n=670)$

\begin{tabular}{|c|c|c|c|c|}
\hline \multirow[t]{2}{*}{ Merkmale } & \multirow{2}{*}{$\begin{array}{l}\text { Total } \\
n=670\end{array}$} & \multirow{2}{*}{$\begin{array}{l}\text { Männlich } \\
n=\mathbf{2 8 7}\end{array}$} & \multirow{2}{*}{$\begin{array}{l}\text { Weiblich } \\
n=294\end{array}$} & \multirow[t]{2}{*}{$p$-Wert } \\
\hline & & & & \\
\hline Alter $(S A)$, in Jahren & $18,80(3,60)$ & $18,23(3,56)$ & $18,59(3,43)$ & 0,21 \\
\hline \multicolumn{5}{|l|}{ Ausbildungslevel der Mutter, $n$ (\%) } \\
\hline Niedrig & $37(5,5)$ & $17(5,9)$ & $15(5,1)$ & \multirow[t]{3}{*}{0,52} \\
\hline Mittel & $405(60,5)$ & $170(59,2)$ & $187(63,8)$ & \\
\hline Hoch & $227(33,9)$ & $100(34,8)$ & $91(31,1)$ & \\
\hline \multicolumn{5}{|l|}{ Ausbildungslevel des Vaters, $n$ (\%) } \\
\hline Niedrig & $30(4,5)$ & $13(4,6)$ & $12(4,2)$ & \multirow[t]{3}{*}{0,96} \\
\hline Mittel & $323(48,7)$ & $141(49,5)$ & $142(49,1)$ & \\
\hline Hoch & $310(46,8)$ & $131(46,0)$ & $135(46,7)$ & \\
\hline Mittleres Stadium, Femur (IQA) & $4(3 a, 4)$ & $4(2 c, 4)$ & $4(3 c, 4)$ & $<0,001$ \\
\hline Mittleres Stadium, Tibia (IQA) & $4(3 b, 4)$ & $4(2 c, 4)$ & $4(3 c, 4)$ & $<0,001$ \\
\hline Mittleres Stadium, Radius (IQA) & $3 c(3 a, 4)$ & $3 c(3 a, 4)$ & $3 c(3 c, 4)$ & 0,005 \\
\hline Mittleres Stadium, Klavikula (IQA) & $3 a(2 a, 3 c)$ & $3 a(1,3 c)$ & $3 a(2 a, 3 c)$ & 0,018 \\
\hline Mittleres Stadium, dritter Molar (IQA) & $F(E, G)$ & $F(E, G)$ & $F(E, G)$ & 0,068 \\
\hline
\end{tabular}

Tab. 3 Ergebnisse der Regressionsanalysen

\begin{tabular}{|c|c|c|c|c|c|c|c|c|c|}
\hline & \multirow[t]{2}{*}{ Merkmal } & \multicolumn{4}{|c|}{ Männlich } & \multicolumn{4}{|c|}{ Weiblich } \\
\hline & & $\beta$ & $95 \%-K I$ & $p$-Wert & $r^{2}$ & $\boldsymbol{\beta}$ & $95 \%-K I$ & $p$-Wert & $r^{2}$ \\
\hline \multirow{6}{*}{$\begin{array}{l}\text { Höchster } \\
\text { Bildungs- } \\
\text { abschluss } \\
\text { der Eltern }\end{array}$} & Alle ossären Merkmale & $-1,36$ & $-3,15 ; 0,42$ & 0,13 & 0,01 & $-0,03$ & $-1,37 ; 1,30$ & 0,96 & 0,00 \\
\hline & Femur & $-0,25$ & $-0,63 ; 0,13$ & 0,19 & 0,01 & $-0,04$ & $-0,35 ; 0,27$ & 0,80 & 0,00 \\
\hline & Tibia & $-0,23$ & $-0,60 ; 0,13$ & 0,21 & 0,01 & $-0,03$ & $-0,31 ; 0,24$ & 0,81 & 0,00 \\
\hline & Radius & $-0,29$ & $-0,63 ; 0,04$ & 0,085 & 0,01 & $-0,09$ & $-0,36 ; 0,17$ & 0,49 & 0,00 \\
\hline & Klavikula & $-0,33$ & $-0,93 ; 0,26$ & 0,27 & 0,01 & 0,06 & $-0,45 ; 0,58$ & 0,80 & 0,00 \\
\hline & Zahn & $-0,12$ & $-0,35 ; 0,10$ & 0,28 & 0,00 & $-0,01$ & $-0,21 ; 0,20$ & 0,95 & 0,00 \\
\hline \multirow{6}{*}{$\begin{array}{l}\text { Gesamter } \\
\text { Bildungs- } \\
\text { abschluss } \\
\text { der Eltern }\end{array}$} & Alle ossären Merkmale & $-0,62$ & $-1,61 ; 0,36$ & 0,22 & 0,01 & 0,01 & $-0,75 ; 0,77$ & 0,97 & 0,00 \\
\hline & Femur & $-0,13$ & $-0,35 ; 0,08$ & 0,23 & 0,01 & $-0,04$ & $-0,22 ; 0,14$ & 0,65 & 0,00 \\
\hline & Tibia & $-0,09$ & $-0,30 ; 0,12$ & 0,41 & 0,00 & $-0,01$ & $-0,17 ; 0,14$ & 0,89 & 0,00 \\
\hline & Radius & $-0,14$ & $-0,33 ; 0,05$ & 0,15 & 0,01 & $-0,06$ & $-0,22 ; 0,09$ & 0,41 & 0,00 \\
\hline & Klavikula & $-0,18$ & $-0,50 ; 0,15$ & 0,29 & 0,01 & 0,10 & $-0,20 ; 0,39$ & 0,52 & 0,00 \\
\hline & Zahn & $-0,10$ & $-0,22 ; 0,02$ & 0,11 & 0,01 & $-0,00$ & $-0,12 ; 0,11$ & 0,99 & 0,00 \\
\hline
\end{tabular}

als aussagekräftigster Parameter im Sinne der Forschungsfrage [26] lag für alle Untersuchungen unter $\mathrm{r}^{2}=0,01$. Dies bedeutet, dass weniger als $1 \%$ der Varianz in den evaluierten Merkmalen auf Unterschiede im SES zurückführbar war.

Die Werte für den Regressionskoeffizienten $(\beta)$ lagen für die Untersuchungen zwischen $\beta=0,10$ und $\beta=-1,36$.

\section{Diskussion}

Insgesamt konnte keine Evidenz für einen Zusammenhang zwischen dem
Bildungsniveau der Eltern und dem Entwicklungsgrad der untersuchten ossären Merkmale festgestellt werden. Dies gilt sowohl für den Ansatz, wenn nur der jeweils höhere Bildungsabschluss der Eltern in die Untersuchung einbezogen wurde, als auch, wenn beide Bildungsabschlüsse der Eltern im Sinne eines Gesamtbildungsniveaus berücksichtigt wurden (•Tab. 3). Es konnte für beide Geschlechter explizit auch kein $\mathrm{Zu}$ sammenhang festgestellt werden, wenn alle untersuchten ossären Merkmale, im
Sinne einer Gesamtskelettentwicklung, zusammengefasst wurden.

Ferner konnte kein Zusammenhang zwischen dem Stadium der Zahnentwicklung und dem Bildungsniveau der Eltern nachgewiesen werden. Damit ist im untersuchten Kollektiv neben der Skelettentwicklung auch die Zahnentwicklung bei beiden Geschlechtern unabhängig vom sozioökonomischen Status (SES). Dieses Ergebnis für die Zahnentwicklung war, im Gegensatz zum Ergebnis für die Skelettentwicklung, 
im Rahmen der Forschungshypothese erwartet worden.

Mit dem Begriff „sozioökonomischer Status", für den synonym auch der Terminus "Sozialstatus" verwendet wird, wird synoptisch die Stellung eines Menschen innerhalb einer Gesellschaft beschrieben. Zur Bestimmung des SES wird zumeist auf Informationen zur schulischen und zur beruflichen Bildung zurückgegriffen. Darüber hinaus können Informationen zur beruflichen Stellung und zur Einkommenssituation berücksichtigt werden [25]. Für die Feststellung des SES von Kindern ist der Ansatz etabliert, auf das Bildungsniveau, die berufliche Stellung, sowie das Einkommen der Eltern zurückzugreifen [27]. Aus Praktikabilitätsgründen wurde in der vorliegenden Studie der Einkommensstatus der Eltern nicht erfasst. Von den Teilnehmern, die älter als 18 Jahre alt waren, wurde nicht der eigene bisher erreichte Bildungsabschluss oder angestrebte Abschluss festgehalten, sondern auch der Bildungsabschluss der Eltern, um die Erfassung einheitlich zu halten.

Für einen Vergleich der Häufigkeiten der Bildungsabschlüsse bei den Eltern der Studienteilnehmer mit der Normalbevölkerung in Deutschland ist zu berücksichtigen, dass in der vorliegenden Studie immer der höchste erreichte Bildungsabschluss angegeben wurde. Die Daten aus den Mikrozensusumfragen, welche vom Statistischen Bundesamt veröffentlicht werden, führen Schulbzw. Berufsabschlüsse getrennt auf [28]. Daher sind insbesondere die Level 2 und 3 der vorliegenden Studie mit den Daten des Statistischen Bundesamtes vergleichbar, da hier die Schulabschlüsse unberücksichtigt bleiben und es nicht zu Doppelzählungen (Schulabschluss und berufliche Bildung) kommt. Nach den Daten des Mikrozensus wiesen etwa 9,5\% der Bevölkerung in Deutschland einen Bildungsabschluss entsprechend Level 3 auf. Etwa $63 \%$ wiesen einen Bildungsabschluss auf, welcher dem Level 2 entspricht [28]. Mit Blick auf die Ergebnisse der vorliegenden Studie fällt auf, dass insbesondere das Level 3, also ein hoher Bildungsabschluss im Sinne von universitären Abschlüssen, deutlich überrepräsentiert ist. Die Studienkohor- te bildet daher nicht die Situation der Normalbevölkerung ab, wobei der SES der Teilnehmer insgesamt höher als der in der Normalbevölkerung ist. In der Gesamtbeurteilung konnte für die Mütter und Väter der Studienteilnehmer bei $40 \%$ ein hohes Bildungslevel festgestellt werden. Insbesondere bei den Vätern der Studienteilnehmer konnte mit $46 \%$ bei fast der Hälfte ein universitärer Abschluss festgestellt werden. Sehr niedrige Bildungsabschlüsse im Sinne von einem fehlenden Schulabschluss bzw. dem Hauptschulabschluss als höchstem erreichten Bildungsabschluss waren insgesamt in unserem Kollektiv nur sehr selten vertreten $(2,3 \%)$. Somit bildet das Studienkollektiv eine Gruppe von Personen mit einem im Vergleich zur Normalbevölkerung in Deutschland höheren SES.

In der Literatur liegen Daten zum Zusammenhang zwischen dem SES und dem Skelettalter vor. Diese Daten stammen überwiegend aus Ländern mit niedrigem und mittlerem Einkommen [15, 29-32]. Im Jahr 2010 untersuchten Chaumoitre et al. den Zusammenhang zwischen dem SES und dem Skelettalter bei 623 Kindern im Alter von 6 bis 18 Jahren in Marrakesch, Marokko [15]. Die Skelettentwicklung wurde nach der Methode von Greulich und Pyle evaluiert. Die Autoren fanden einen signifikanten Zusammenhang zwischen SES und Skelettalter, wobei ein niedriger SES mit einem niedrigeren Skelettalter assoziiert war. Chaumoitre et al. definierten in ihrer Studie den SES in 2 Kategorien: privilegiert und nichtprivilegiert. Dabei wurden folgende Aspekte berücksichtigt: Beruf der Eltern, Bildungsniveau der Eltern, Fähigkeit der Eltern, zu lesen und zu schreiben, Haushaltsgröße, Zusammensetzung der Familie. Bei den Kriterien der Autoren für den SES fällt auf, dass diese nicht direkt mit den Kriterien der vorliegenden Studie zu vergleichen sind. Es ist davon auszugehen, dass die Unterschiede in den Lebensumständen zwischen den „Privilegierten“ und den "Nichtprivilegierten" deutlich höher sein dürften als die Unterschiede zwischen den Leveln 1 und 3 in der vorliegenden Studie.

Ein Zusammenhang zwischen dem SES und dem Skelettalter konnte auch in
Ländern mit niedrigem und mittlerem Einkommen festgestellt werden. Dabei war ein relativ niedriger SES immer mit einer zurückgebliebenen Skelettentwicklung verbunden. Die Methodik der Erfassung des SES sowie die Altersspanne der Kohorten variiert jedoch deutlich [29-31]. So rekrutierten Linhares et al. etwa ihr Kollektiv in Brasilien im Jahr 1986 an Kindern, mit denen sie beim Schuheputzen oder Betteln in Kontakt kamen. Auch hier wird deutlich, dass ein derart niedriges Level beim SES in der vorliegenden Studie nicht abgebildet ist.

In Bezug auf die Altersspanne des untersuchten Kollektivs ist festzustellen, dass beispielsweise Elamin et al. im Jahr 2017 im Nordsudan ein Kollektiv im Alter vom 3 bis 25 Jahren untersuchten. Damit sind in dieser Studie auch wesentlich jüngere Kinder als in der vorliegenden Arbeit untersucht worden [30]. Den SES ermittelten Elamin et al. über die Art der Krankenversicherung bzw. über die Art der besuchten Schule (staatlich oder privat) [30]. Es kann geschlussfolgert werden, dass der SES nur dann Einfluss auf das Skelettalter nimmt, wenn sich die Lebensumstände zwischen den einzelnen SES-Leveln auch tatsächlich deutlich unterscheiden. Von einer solchen Unterscheidung der Lebensumstände bei den Teilnehmern ist für die vorliegende Studie nicht auszugehen. In der $\mathrm{Zu}$ sammenschau wird zudem deutlich, wie heterogen zum einen die Kollektive in den unterschiedlichen Studien sind, v. a. aber auch, wie unterschiedlich der SES ermittelt wurde. Für zukünftige Studien ist eine einheitliche Erfassung des SES, z. B. nach den spezifischen Kriterien der KiGGS-Studie [27], wünschenswert.

Für Länder mit hohem Einkommen liegen aktuelle Daten von Alshamrani und Offiah (2020) vor [33]. Die Autoren untersuchten 2 unterschiedliche Stadiensysteme für die Handskelettentwicklung an einer englischen Population unterschiedlicher Ethnien im Alter von 2 bis 15 Jahren in der Region Sheffield. Der SES wurde über die jeweilige Postleitzahl ermittelt: Über die Postleitzahl wurde per Abgleich mit einer entsprechenden Datenbank („,index of multiple deprivation“) auf den SES rückgeschlossen. Dieser An- 
satz ist grundsätzlich kritisch zu sehen, da keine Angaben über den tatsächlichen SES der Studienteilnehmer vorlagen. Alshamrani und Offiah konnten nur für eine der beiden untersuchten Methoden einen Zusammenhang zwischen Skelettalter und SES feststellen. Für die Methode, welche insgesamt besser mit dem chronologischen Alter der Teilnehmer korrelierte, konnte kein Zusammenhang festgestellt werden [33]. Diese Ergebnisse können - ebenso wie die Resultate der gegenständlichen Studie - als Indiz dafür gewertet werden, dass in Ländern mit geringerem sozialen Gefälle kein offensichtlicher Zusammenhang zwischen SES und Skelettalter festzustellen ist.

\section{Fazit für die Praxis}

\section{- Der sozioökonomische Status (SES) der untersuchten Studienpopulation war höher als in der Allgemeinbevöl- kerung in Deutschland. \\ - Für die Studienpopulation wurde kein Zusammenhang zwischen dem SES und der Skelett- oder Zahnentwicklung gefunden. \\ - Offenbar unterschieden sich die Lebensumstände der Studienteil- nehmer nicht ausreichend stark, um einen Einfluss des SES auf das Skelettalter darzustellen.}

\section{Korrespondenzadresse}

\section{Dr. med. Dr. med. dent. M. Timme}

Institut für Rechtsmedizin, Universitätsklinikum Münster, Westfälische Wilhelms-Universität Münster

Röntgenstraße 23, 48149 Münster, Deutschland maximilian.timme@ukmuenster.de

Funding. Open Access funding enabled and organized by Projekt DEAL.

\section{Einhaltung ethischer Richtlinien}

Interessenkonflikt. M. Timme, A. Karch, D. Shay, C. Ottow und A. Schmeling geben an, dass kein Interessenkonflikt besteht.
Alle beschriebenen Untersuchungen am Menschen oder an menschlichem Gewebe wurden mit Zustimmung der zuständigen Ethikkommission (Ethik-Kommission der Ärztekammer Westfalen-Lippe und der Westfälischen Wilhelms-Universität Münster), im Einklang mit nationalem Recht sowie gemäß der Deklaration von Helsinki von 1975 (in der aktuellen, überarbeiteten Fassung) durchgeführt. Von allen beteiligten Patienten liegt eine Einverständniserklärung vor.

Open Access. Dieser Artikel wird unter der Creative Commons Namensnennung 4.0 International Lizenz veröffentlicht, welche die Nutzung, Vervielfältigung, Bearbeitung, Verbreitung und Wiedergabe in jeglichem Medium und Format erlaubt, sofern Sie den/die ursprünglichen Autor(en) und die Quelle ordnungsgemäß nennen, einen Link zur Creative Commons Lizenz beifügen und angeben, ob Änderungen vorgenommen wurden.

Die in diesem Artikel enthaltenen Bilder und sonstiges Drittmaterial unterliegen ebenfalls der genannten Creative Commons Lizenz, sofern sich aus der Abbildungslegende nichts anderes ergibt. Sofern das betreffende Material nicht unter der genannten Creative Commons Lizenz steht und die betreffende Handlung nicht nach gesetzlichen Vorschriften erlaubt ist, ist für die oben aufgeführten Weiterverwendungen des Materials die Einwilligung des jeweiligen Rechteinhabers einzuholen.

Weitere Details zur Lizenz entnehmen Sie bitte der Lizenzinformation auf http://creativecommons.org/ licenses/by/4.0/deed.de.

\section{Literatur}

1. Marroquin TY, Karkhanis S, Kvaal SI, Vasudavan S, Kruger E, Tennant M (2017) Age estimation in adults by dental imaging assessment systematic review. Forensic Sci Int 275:203-211. https://doi. org/10.1016/j.forsciint.2017.03.007

2. Guo Y-C, Wang Y-H, Olze A, Schmidt S, Schulz R, Pfeiffer H, Chen T, Schmeling A (2020) Dental age estimation based on the radiographic visibility of the periodontal ligament in the lower third molars: application of a new stage classification. Int J Legal Med 134:369-374. https://doi.org/10.1007/ s00414-019-02178-y

3. De Tobel J, van Wijk M, Alberink I, Hillewig E, Phlypo I, van Rijn RR, Thevissen PW, Verstraete KL de Haas MB (2020) The influence of motion artefacts on magnetic resonance imaging of the clavicles for age estimation. Int J Legal Med 134:753-768. https://doi.org/10.1007/s00414019-02230-x

4. Schmeling A, Dettmeyer R, Rudolf E, Vieth V, Geserick G (2016) Forensic Age estimation. Dtsch Arztebl Int 113:44-50. https://doi.org/10.3238/ arztebl.2016.0044

5. De Tobel J, Bauwens J, Parmentier GIL, Franco A, Pauwels NS, Verstraete KL, Thevissen PW (2020) Magnetic resonance imaging for forensic age estimation in living children and young adults: a systematic review. Pediatr Radiol. https://doi. org/10.1007/s00247-020-04709-x

6. Bjørk MB, Kvaal SI (2018) CT and MR imaging used in age estimation: a systematic review. J Forensic Odontostomatol 36:14-25

7. Tozakidou M, Apine I, Petersen KU, Weinrich JM, SchinderaS, Jopp-van WellE, PüschelK, Herrmann J
(2020) Comparison of different iterative CT reconstruction techniques and filtered back projection for assessment of the medial clavicular epiphysis in forensic age estimation. Int J Legal Med 134:355-361. https://doi.org/10.1007/ s00414-019-02214-x

8. Dubourg O, Faruch-Bilfeld M, Telmon N, Savall F, Saint-Martin P (2020) Technical note: age estimation by using pubic bone densitometry according to a twofold mode of CT measurement. Int J Legal Med. https://doi.org/10.1007/s00414-02002349-2

9. Lu T, Shi L, Zhan M-J, Fan F, Peng Z, Zhang K, Deng Z-H (2020) Age estimation based on magnetic resonance imaging of the ankle joint in a modern Chinese Han population. Int J Legal Med 134:1843-1852. https://doi.org/10.1007/s00414020-02364-3

10. Schmeling A, Grundmann C, Fuhrmann A Kaatsch H-J, Knell B, Ramsthaler F, Reisinger W, Riepert T, Ritz-Timme S, Rösing FW, Rötzscher K, Geserick G (2008) Criteria for age estimation in living individuals. Int J Legal Med 122:457-460. https://doi.org/10.1007/s00414-008-0254-2

11. Wittschieber $D$, Ottow $C$, Vieth V, Küppers $M$, Schulz R, Hassu J, Bajanowski T, Püschel K, Ramsthaler F, Pfeiffer H, Schmidt S, Schmeling A (2015) Projection radiography of the clavicle: still recommendable for forensic age diagnostics in living individuals? Int J Legal Med 129:187-193. https://doi.org/10.1007/s00414-014-1067-0

12. Bedek I, Dumančić J, Lauc T, Marušić M, ČukovićBagić I (2020) New model for dental age estimation: Willems method applied on fewer than seven mandibular teeth. Int J Legal Med 134:735-743. https://doi.org/10.1007/s00414019-02066-5

13. Timme M, Karch A, Shay D, Ottow C, Schmeling A (2020) The relevance of body mass index in forensic age assessment of living individuals: an age-adjusted linear regression analysis using multivariable fractional polynomials. Int J Legal Med 134:1861-1868. https://doi.org/10.1007/ s00414-020-02381-2

14. Schmeling A, Reisinger W, Loreck D, Vendura K, Markus W, Geserick G (2000) Effects of ethnicity on skeletal maturation: consequences for forensicage estimations. Int J Legal Med 113:253-258. https:// doi.org/10.1007/s004149900102

15. Chaumoitre K, Lamtali S, Baali A, Saliba-Serre B, Lahmam A, Aboussad A, Boëtsch G, Panuel M (2010) Influence of Socioeconomic Status and Body Mass Index on Bone Age. Horm Res Paediatr 74:129-135. https://doi.org/10.1159/000313371

16. Pelsmaekers B, Loos R, Carels C, Derom C, Vlietinck R (1997) The genetic contribution to dental maturation. J Dent Res 76:1337-1340. https://doi.org/10.1177/00220345970760070201

17. Olze A, Schmeling $A$, Taniguchi $M$, Maeda $H$, van NiekerkP, Wernecke K-D, Geserick G (2004) Forensic age estimation in living subjects: the ethnic factor in wisdom tooth mineralization. Int J Legal Med 118:170-173. https://doi.org/10.1007/s00414004-0434-7

18. Olze A, van Niekerk P, Ishikawa T, Zhu BL, Schulz R, Maeda H, Schmeling A (2007) Comparative study on the effect of ethnicity on wisdom tooth eruption. Int J Legal Med 121:445-448. https://doi. org/10.1007/s00414-007-0171-9

19. Olze A, Reisinger W, Geserick G, Schmeling A (2006) Age estimation of unaccompanied minors. Part II. Dental aspects. Forensic Sci Int 159(Suppl 1):S65-S67. https://doi.org/10.1016/j.forsciint. 2006.02.018 
20. Schmeling A, Schulz R, Reisinger W, Mühler M, Wernecke K-D, Geserick G (2004) Studies on the time frame for ossification of the medial clavicular epiphyseal cartilage in conventional radiography. Int J Legal Med 118:5-8. https://doi.org/10.1007/ s00414-003-0404-5

21. Kellinghaus $M$, Schulz R, Vieth $V$, Schmidt $S$, Pfeiffer $\mathrm{H}$, Schmeling A (2010) Enhanced possibilities to make statements on the ossification status of the medial clavicular epiphysis using an amplified staging scheme in evaluating thin-slice CT scans. Int J Legal Med 124:321-325. https://doi.org/10. 1007/s00414-010-0448-2

22. Demirjian A, Goldstein H, Tanner JM (1973) A new system of dental age assessment. Hum Biol 45:211-227

23. Mincer HH, Harris EF, Berryman HE (1993) The A.B.F.O. study of third molar development and its use as an estimator of chronological age. J Forensic Sci 38:379-390

24. UNESCO Institute for Statistics (2012) Internationa standard classification of education: ISCED 2011. UNESCO Institute for Statistics, Montreal

25. Lampert T, Kroll LE (2006) Messung des sozioökonomischen Status in sozialepidemiologischen Studien. In: Richter M, Hurrelmann K (Hrsg) Gesundheitliche Ungleichheit. VS, Wiesbaden

26. Backhaus K, Erichson B, Plinke W, Weiber R (2018) Multivariate Analysemethoden: eine anwendungsorientierte Einführung, 15. Aufl. Springer Gabler, Berlin Heidelberg

27. KiGGS Study Group, Lampert T, Müters S, Stolzenberg H, Kroll LE (2014) Messung des sozioökonomischen Status in der KiGGS-Studie: Erste Folgebefragung (KiGGS Welle 1). Bundesgesundheitsblatt Gesundheitsforschung Gesundheitsschutz 57:762-770. https://doi.org/ 10.1007/s00103-014-1974-8

28. Statistisches Bundesamt Bevölkerung nach Bildungsabschluss in Deutschland. https://www. destatis.de/DE/Themen/Gesellschaft-Umwelt/ Bildung-Forschung-Kultur/Bildungsstand/ Tabellen/bildungsabschluss.html.Zugegriffen: 26 . Aug. 2020

29. Linhares ED, Round JM, Jones DA (1986) Growth, bone maturation, and biochemical changes in Brazilian children from two different socioeconomic groups. Am J Clin Nutr 44:552-558. https://doi. org/10.1093/ajcn/44.4.552

30. Elamin F, Abdelazeem N, Elamin A, Saif D, Liversidge HM (2017) Skeletal maturity of the hand in an East African group from Sudan. Am J Phys Anthropol 163:816-823. https://doi.org/10.1002/ ajpa.23247

31. Bogin B, MacVean RB (1983) The relationship of socioeconomic status and sex to body size, skeletal maturation, and cognitive status of Guatemala City schoolchildren. Child Dev 54:115-128

32. Bundesministerium für wirtschaftliche Zusammenarbeit und Entwicklung DAC-Liste der Entwicklungsländer und-gebiete. https://www.bmz. de/de/zentrales_downloadarchiv/Ministerium/ ODA/DAC_Laenderliste_Berichtsjahre_2018_ 2020.pdf.Zugegriffen: 26. Aug. 2020

33. Alshamrani K, Offiah AC (2020) Applicability of two commonly used bone age assessment methods to twenty-first century UK children. Eur Radiol 30:504-513. https://doi.org/10.1007/ s00330-019-06300-x

\section{Lesen Sie Ihre Zeitschrift online auf SpringerMedizin.de}

SpringerMedizin.de bietet Ihnen Zugang zu allen elektronisch verfügbaren Ausgaben und dem CME-Angebot Ihrer Zeitschrift - unabhängig davon, seit wann Sie die Zeitschrift abonniert haben.

So einfach erhalten Sie Zugang zum Online-Archiv:

- Registrieren Sie sich einmalig auf www.springermedizin.de/register

Geben Sie dabei Ihre Einheitliche Fortbildungsnummer (EFN) an.

- Ihr Benutzername entspricht Ihrer E-Mail-Adresse, Ihr Passwort können Sie frei wählen und später jederzeit unter "Mein Profil" ändern.

- Falls Sie bereits ein (Print-) Abonnement bei uns haben, geben Sie bei der Registrierung die Lieferadresse Ihrer Zeitschrift an. Damit wird Ihr Abo-Zugang auf springermedizin.de freigeschaltet.

Sind Sie bereits bei SpringerMedizin.de registriert?

Dann wird Ihr Zeitschriftenabonnement automatisch Ihrem Online-Nutzerkonto hinzugefügt. Sollten die Angaben Ihres Online-Accounts nicht eindeutig mit den Angaben Ihres Zeitschriften-Abonnements übereinstimmen, kann die Zuordnung nicht sicher erfolgen. In diesem Fall und bei allen anderen Fragen zum Online-Zugang kontaktieren Sie bitte unseren Kundenservice unter: Kundenservice@springermedizin.de

Telefonisch erreichen Sie die Hotline montags bis freitags von 9.00 bis $17.00 \mathrm{Uhr}$ kostenfrei unter 0800-77 80777 sowie gebührenpflichtig aus dem Ausland unter +4930884293600. 